\title{
CHAOTIC WALKING AND FRACTAL SCATTERING OF ATOMS IN A TILTED OPTICAL LATTICE
}

\author{
S.V. Prants, V.O. Vitkovsky \\ Laboratory of Nonlinear Dynamical Systems, \\ Pacific Oceanological Institute of the Russian Academy of Sciences, \\ 690041 Vladivostok, Russia, URL: dynalab.poi.dvo.ru \\ *Corresponding author e-mail: prants@poi.dvo.ru
}

\begin{abstract}
Chaotic walking of cold atoms in a tilted optical lattice, created by two counter propagating running waves with an additional external field, is demonstrated theoretically and numerically in the semiclassical and Hamiltonian approximations. The effect consists in random-like changing the direction of atomic motion in a rigid lattice under the influence of a constant force due to a specific behavior of the atomic dipole-moment component that changes abruptly in a random-like manner while atoms cross standing-wave nodes. Chaotic walking generates a fractal-like scattering of atoms that manifests itself in a self-similar structure of the scattering function in the atom-field detuning, position and momentum spaces. The probability distribution function of the scattering time is shown to decay in a non-exponential way with a power-law tail.
\end{abstract}

Keywords: cold atom, tilted potential, chaos, fractal

\section{Introduction}

The mechanical action of light upon neutral atoms placed in a laser standing wave is at the heart of laser cooling, trapping, and Bose-Einstein condensation [1]. Numerous applications of the mechanical action of light include isotope separation, atomic interferometry, atomic lithography and epitaxy, atomic-beam deflection and splitting, manipulating translational and internal atomic states, measurement of atomic positions, etc. Atoms and ions in an optical lattice, formed by a laser standing wave, are perspective objects for implementation of quantum information processing and quantum computing. Advances in cooling and trapping of atoms, tailoring optical potentials of a desired form and dimension, controlling the level of dissipation and noise are now enabling the direct experiments with single atoms to study fundamental principles of quantum physics, quantum chaos, decoherence, and quantum-classical correspondence.

Nonlinear dynamics of cold atoms in optical lattices is a fastly growing branch of atomic physics. There are a number of theoretical works and impressive experiments on quantum chaos, dynamical localization, chaos-assisted tunneling, Lévy flights, etc. (for reviews see [2,3]). To suppress spontaneous 
emission and provide a coherent quantum dynamics one usually works far from the optical resonance. Adiabatic elimination of the excited state amplitude leads to an effective Hamiltonian for the centerof-mass motion, whose 3/2 degree-of-freedom classical analogue has a mixed phase space with regular islands embedded in a chaotic sea. New possibilities are opened if one works near the optical resonance and take the internal atomic dynamics into account. A single atom in a standing-wave laser field may be semiclassically treated as a nonlinear dynamical system with coupled internal (electronic) and external (mechanical) degrees of freedom [4-6]. In the semiclassical and Hamiltonian limits (when one treats atoms as point-like particles and neglects spontaneous emission and other losses of energy), a number of nonlinear dynamical effects have been analytically and numerically demonstrated with this system: chaotic Rabi oscillations [4 6], Hamiltonian chaotic atomic transport and dynamical fractals [7-10], Lévy flights and anomalous diffusion [6,11,12. These effects are caused by local instability of the CM motion in a laser field. A set of atomic trajectories under certain conditions becomes exponentially sensitive to small variations in initial quantum internal and classical external states or/and in the control parameters, mainly, the atom-laser detuning. Hamiltonian evolution is a smooth process that is well described in a semiclassical approximation by the coupled Hamilton-Schrödinger equations. A detailed theory of Hamiltonian and dissipative chaotic transport of atoms in a laser standing wave has been developed in Refs. [10] and [13, 14, respectively.

Additional possibilities to manipulate the atomic transport are created by applying an external force to the standing-wave optical potential. It is obvious that for cold atoms in a vertical optical lattice it is necessary to account for the Earth's acceleration. It is possible as well to create horizontal accelerated optical lattices by adding a constant force whose magnitude along the optical axis can be easily varied. The problem of atomic motion in a tilted optical potential is closely related to the old problem of electron motion in a a periodic crystal with dc or ac forces applied. The analogue of well known Bloch oscillations with cold atoms has been experimentally found in Ref. [15, 16].

In the present paper we apply the ideas and methods, elaborated in the field of nonlinear dynamics of cold atoms, to study theoretically and numerically motion of point-like atoms in a tilted optical lattice. It will be shown that varying only one parameter, the detuning between the frequencies of a working atomic transition and the laser field, one can explore a variety of regimes of atom motion, including chaotic walking, dynamical fractals and chaotic scattering.

\section{Chaotic and regular regimes of motion of atoms in a tilted potential}

In the one-dimensional case, the Hamiltonian of a two-level atom in a standing-wave laser field and an additional external field can be written in the frame rotating with the laser frequency $\omega_{f}$ as follows:

$$
\hat{H}=\frac{P^{2}}{2 m_{a}}+\frac{\hbar}{2}\left(\omega_{a}-\omega_{f}\right) \hat{\sigma}_{z}-\hbar \Omega_{0}\left(\hat{\sigma}_{-}+\hat{\sigma}_{+}\right) \cos k_{f} X+F X
$$

where $\hat{\sigma}_{ \pm, z}$ are the Pauli operators for the internal atomic degrees of freedom, $X$ and $P$ are the classical atomic position and momentum, $\omega_{a}$ and $\Omega_{0}$ are the atomic transition and maximal Rabi frequencies, respectively. $F$ stands for the static force induced by external field.

In the semiclassical approximation, where the transversal atomic momentum $p$ is supposed to be, in average, much larger than the photon one $\hbar k_{f}$, atom with quantized internal dynamics is treated as a 
point-like particle to be described by the Bloch-Hamilton equations of motion without relaxation terms

$$
\begin{gathered}
\dot{x}=\omega_{r} p, \quad \dot{p}=-u \sin x-\kappa, \quad \dot{u}=\Delta v, \\
\dot{v}=-\Delta u+2 z \cos x, \quad \dot{z}=-2 v \cos x,
\end{gathered}
$$

where $u$ and $v$ are synchronized (with the laser field) and quadrature components of the atomic electric dipole moment, respectively, and $z$ is the atomic population inversion. Equations (2) are written in the dimensionless form with $x \equiv k_{f} X$ and $p \equiv P / \hbar k_{f}$ to be classical atomic center-of-mass position and momentum, respectively. Dot denotes differentiation with respect to the dimensionless time $\tau \equiv \Omega t$. The set (2) has the three control parameters

$$
\omega_{r} \equiv \hbar k_{f}^{2} / m_{a} \Omega, \quad \Delta \equiv\left(\omega_{f}-\omega_{a}\right) / \Omega, \quad \kappa \equiv F / \hbar k_{f} \Omega,
$$

which are the normalized recoil frequency, $\omega_{r}$, atom-field detuning, $\Delta$, and applied force $\kappa$. The system has two integrals of motion, namely the total energy

$$
H \equiv \frac{\omega_{r}}{2} p^{2}+\kappa x-u \cos x-\frac{\Delta}{2} z,
$$

and the length of the Bloch vector $u^{2}+v^{2}+z^{2}=1$.

The external force is directed in the negative direction of the optical axis $x$. So, if the initial atomic momentum, $p_{0}$, is chosen to be in the negative direction, the force will simply accelerate the corresponding atoms. If $p_{0}>0$, then one may expect much more complicated motion.

Equations (2) constitute a nonlinear Hamiltonian autonomous system with two and half degrees of freedom. Owing to two integrals of motion, phase points move on a three-dimensional hypersurface with a given energy value $H$. In general, motion in a three-dimensional phase space in characterized by a positive Lyapunov exponent, a negative exponent equal in magnitude to the positive one, and zero exponent. The maximal Lyapunov exponent characterizes the mean rate of the exponential divergence of initially close trajectories and serves as a quantitative measure of dynamical chaos in the system. Because of a transient character of chaos, we have computed the finite-time Lyapunov exponent $\lambda$ by the method developed in Refs. [17, 18]. It has been found that at the fixed value, $\omega_{r}=10^{-3}$, of the recoil frequency $\lambda$ is positive in the following ranges of values of the other control parameters: the detuning $-0.5<\Delta<0.5$ and the force $-0.25<\kappa<0.6$. Therefore, we expect chaotic motion of atoms with the parameter's values in those ranges.

In numerical experiments throughout the paper we suppose that two-level atoms are initially prepared in the ground states, $u_{0}=v_{0}=0, z_{0}=-1$, with $x_{0}=0$ and fixed values of the two control parameters, the normalized recoil frequency, $\omega_{r}=10^{-3}$, and the external force $\kappa=0.01$. The atom-field detuning, $\Delta$, can be changed in a wide range. It will be shown in this paper that atoms may perform chaotic walking, the new type of motion in absolutely deterministic environment where atoms can change the direction of motion alternating between flying through the standing wave and being trapped in its potential wells. We would like to stress that the local instability produces chaotic center-of-mass motion in a rigid optical lattice without any modulation of its parameters.

In Fig. 1 1we illustrate different regimes of the center-of-mass atomic motion along the optical axis with the initial atomic momentum chosen to be $p_{0}=10$. It is simply the motion on the phase plane $x-p$. A typical picture with chaotic walking is shown in Fig. 1 a with the value of the detuning, $\Delta=0.15$, at which the maximal Lyapunov exponent, $\lambda$, is positive. The atom starts to move in the positive $x$-direction, 
changes soon the direction of motion a few times, acquiring irregularly positive and negative values of the momentum, and suddenly begins to move in the positive $x$-direction for a comparatively long time. Then it is decelerated, turns back and flies in the negative direction. After that it changes the direction of motion many times demonstrating what we call "chaotic walking".

For comparison, we show in Fig. 1b the phase-plane motion with the larger detuning $\Delta=1$ (and at the same other conditions) at which the maximal Lyapunov exponent is not positive. The atom moves initially in the positive $x$-direction, accelerating and decelerating alternatively. Soon it changes the direction of motion and moves permanently in the negative direction. The motion is regular with a slight modulation of the momentum. The motion at exact resonance, $\Delta=0$, is even more simple (Fig. 17).

What is the ultimate reason of chaotic walking? For an optical lattice without an external force, it has been found in Ref. [10] that instability is caused by the specific behavior of the Bloch-vector component of a moving atom, $u$, whose shallow oscillations between the standing-wave nodes are interrupted by sudden jumps with different amplitudes while atom crosses each node of the wave. It looks like a random like shots happened in a fully deterministic environment. The reason of chaotic walking in a tilted potential is the same. It follows from the second equation in the set (2) that those jumps result in sudden changes of the atomic momentum while crossing nodes. If the value of the atomic energy is close to the separatrix one, the atom after the corresponding jump-like change in $p$ can either overcome the potential barrier and leave a potential well or it will be trapped by the well, or it will move as before crossing the node. The evolution of all the Bloch components in the regime of chaotic walking is shown in Fig. 2, For comparison, we show in Figs. 3 and 4 their evolution in the regular regimes, far off the resonance and at exact resonance, respectively.

\section{Fractal scattering of atoms in a tilted potential}

Different types of fractal-like structures may arise in chaotic Hamiltonian systems (see reviews [19]20]). It is known from many studies in celestial mechanics [21], fluid dynamics [22, 23, atomic physics [7, 10, 12, 25], cavity quantum electrodynamics [8, 9], underwater acoustics [24] and other disciplines [26] that under certain conditions the motion inside an interaction region may have features that are typical for dynamical chaos, (homoclinic and heteroclinic tangles, fractals, strange invariant sets, positive finite-time Lyapunov exponents, etc.) although the particle's trajectories are not chaotic in a rigorous sense because chaos is defined as an irregular motion over infinite time.

Let us place atoms one by one at the point $x_{0}=0$ with the same value of the initial momentum $p_{0}=10$ but change slightly the value of the detuning $\Delta$. All the other initial conditions and the control parameters are supposed to be the same for all the atoms. We fix the time moment $T$ when each atom crosses the point $x=0$ moving in the negative direction. The exit time function $T(\Delta)$ in Fig. 5 demonstrates the complicated structure with smooth intervals alternating with wildly oscillating peaks that cannot be resolved in principle, no matter how large the magnification factor. The panels (b) and (c) in Fig. [5] are successive 50 times magnifications of the detuning intervals shown in the panel (a). Further magnifications reveal a self-similar fractal-like structure that is typical for Hamiltonian systems with chaotic scattering. The exit time $T$ increases with increasing the magnification factor. The same picture is observed when computing the exit time function in the position and momentum spaces. It is a clear demonstration of a fractal-like behavior of chaotically walking atoms. 
It is established in theory of one and half degree-of-freedom systems that transient Hamiltonian chaos in the interaction region occurs due to existence of, at least, one non-attractive chaotic invariant set consisting of an infinite number of localized unstable periodic orbits and aperiodic orbits. This set possesses stable and unstable manifolds extending into the regions of regular motion. The particles with the initial positions close to the stable manifold follow the chaotic-set trajectories for a comparatively long time, then deviate from them, and leave the interaction region along the unstable manifold. In a typical Hamiltonian system there exists an infinite number of trajectories of zero measure with infinite exit time which belong to that chaotic invariant set. Our system with two and half degrees of freedom is a much more complicated one, and it is practically impossible to reveal the corresponding chaotic invariant set with its stable and unstable manifolds. However, the mechanism of chaotic scattering and fractal-like structures should be the same.

The statistics of exit times $T$ is shown in Fig. 6 in a semilogarithmic and logarithmic scales. The probability distribution function (PDF) in this figure gives the probability for an atom to have a given value of $T$. The bold straight line in Fig. 6a implies that the PDF is exponential in its middle part, $P \sim \exp (-\alpha T)$, with the exponent $\alpha=-0.000270722$. However, the tail of the PDF is not exponential. To prove that we plot the function in the logarithmic scale in Fig. 6b and compute the slope at the tail. It has no sense to calculate the slope at the very tail because of a small number of events with very large values of $T$. The bold straight line implies that the PDF is a power-law one, $P \sim T^{-\gamma}$, with the coefficient $\gamma=-2.53086$. It is interesting that the slope at the PDF tail around the value -2.5 is rather typical for many chaotic Hamiltonian systems [27,28]. The reason of that is unclear.

In hyperbolic chaotic systems the PDFs should decay exponentially because the phase space of such systems is homogeneous, and all the trajectories are unstable. It is not the case even with one and half degree-of-freedom systems with inhomogeneous phase space, where exist so-called stability islands embedded in a stochastic sea, because the borders of those islands are "sticky". It means that a typical chaotic trajectory, wandering in the stochastic sea, approaches the island's borders and "stick" to them for a long time. By that reason, the corresponding PDFs are not exponential but power-law ones at their tails. PDFs with power-law decay imply that the corresponding quantity, the exit time in our case, is scale invariant i.e., there is no a single dominant scale in the process. Geometrically it means that chaotic trajectories for such a process are self-similar.

\section{Conclusion}

It is shown that point-like atoms in a tilted optical potential with a constant external force applied can move chaotically changing the direction of motion in a random-like way. The existence of chaos is confirmed by direct computation of the maximal finite-time Lyapunov exponent of the equations of motion that is shown to be positive in a range of the atom-laser detuning and the applied-force strength. The ultimate reason of chaotic walking is the specific behavior of the Bloch-vector component of a moving atom, $u$, whose shallow oscillations between the standing-wave nodes are interrupted by sudden jumps with different amplitudes while atom crosses each node of the wave. It is demonstrated numerically that such a behavior arises exactly at those values of the detuning for which the Lyapunov exponent is positive and atoms move chaotically. We illustrate different regimes of the center-of-mass motion simply varying the detuning. It is an easy way to manipulate the atomic transport in tilted optical lattices.

Treating motion of atoms in a tilted optical lattice as a scattering problem, we show that the scattering 

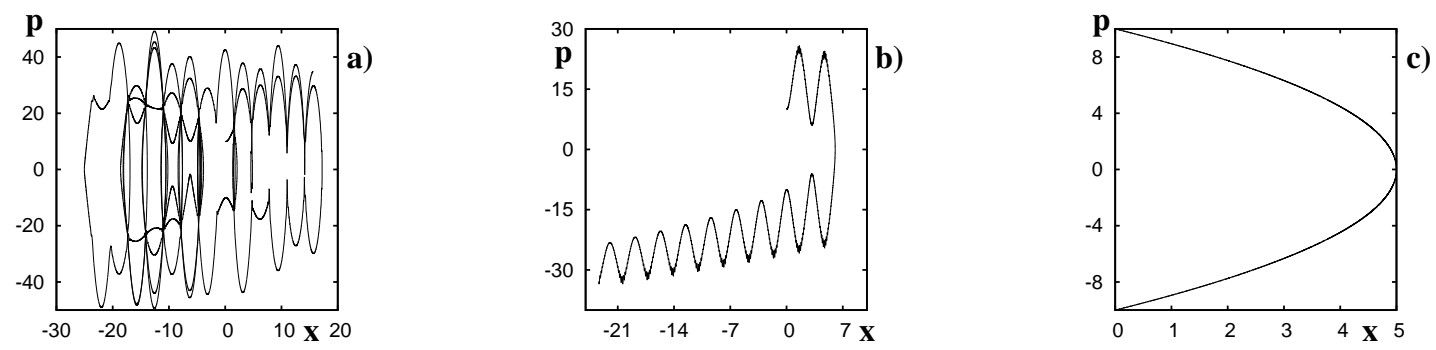

Figure 1: Motion of a cold atom in a deterministic tilted optical lattice as it looks on the phase plane $x-p$. (a) Chaotic walking at $\Delta=0.15, \kappa=0.01, \omega_{r}=10^{-3}$. (b) Regular motion at $\Delta=1$ and with the same other conditions. (c) Regular motion at the resonance, $\Delta=0$, with the same other conditions.
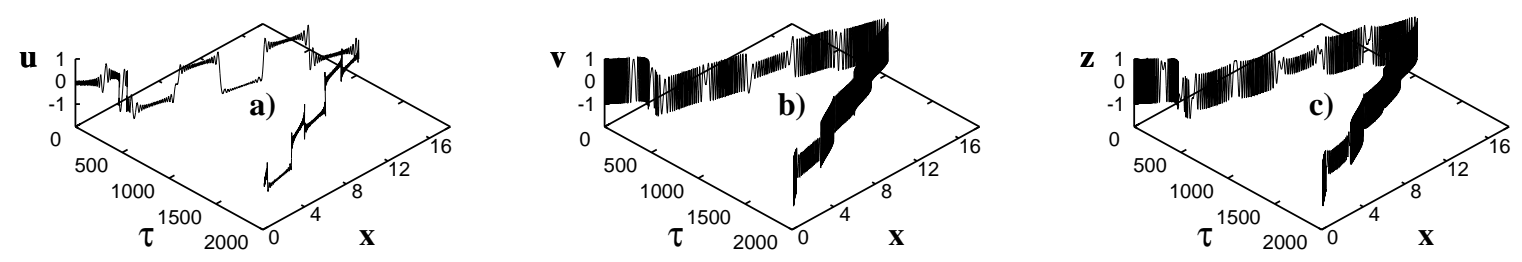

Figure 2: Evolution of the atomic Bloch components in the regime of chaotic walking $(\Delta=0.15)$.

of atoms under conditions of chaotic walking is chaotic and typical for Hamiltonian systems. Fixing the time moment $T$ when atoms with slightly different values of the detuning, momentum or initial position cross a fixed point $(x=0)$, we show that the corresponding scattering functions demonstrate the complicated structure that cannot be resolved in principle, no matter how large the magnification factor. Owing to that the probability to have a given value of $T$ is not exponential but decays at its tail by a power law.

\section{Acknowledgments}

This work was supported by the Integration grant from the Far-Eastern and Siberian branches of the Russian Academy of Sciences (12-II-0-02-001), and by the Program "Fundamental Problems of Nonlinear Dynamics in Mathematics and Physics".

\section{References}

[1] S. Chu, Rev. Mod. Phys., 73, 685 (1998); C. Cohen-Tannoudji, ibid, 707 (1998); W.D. Phillips, ibid, 721 (1998).

[2] M. G. Raizen, Adv. At. Mol. Opt. Phys., 41, 43 (1999). 

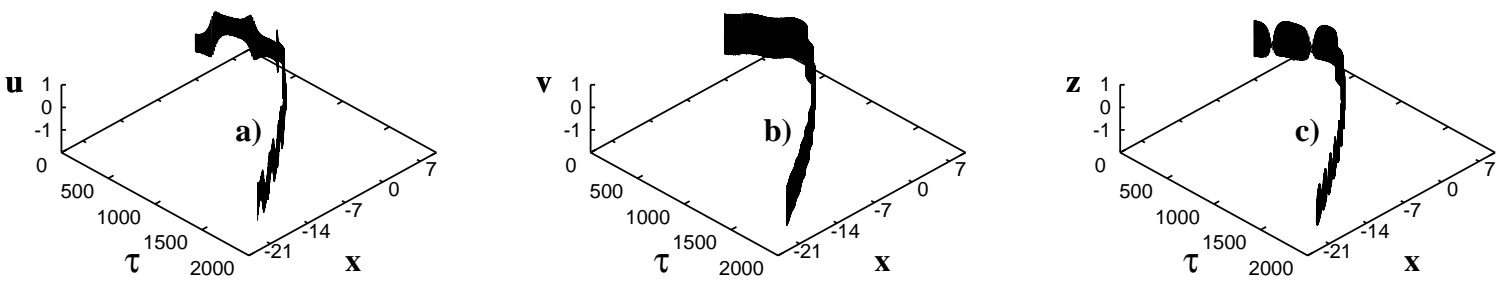

Figure 3: The same as in Fig. 2 but far from the resonance $(\Delta=1)$.
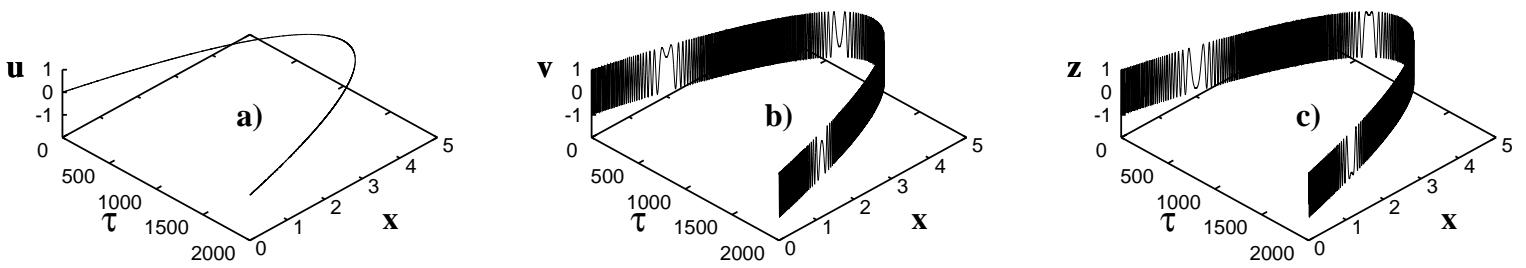

Figure 4: The same as in Fig. 2 but at the resonance $(\Delta=0)$.
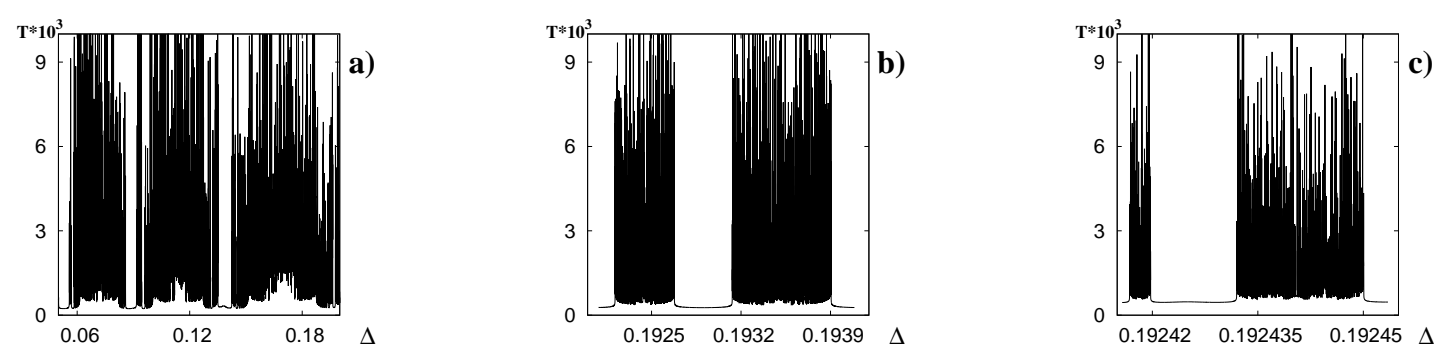

Figure 5: Atomic dynamical fractal. Self-similar dependence of the exit time, $T$, with given initial position, $x_{0}=0$ and momentum $p_{0}=10$, on the detuning. The successive magnifications are shown. 

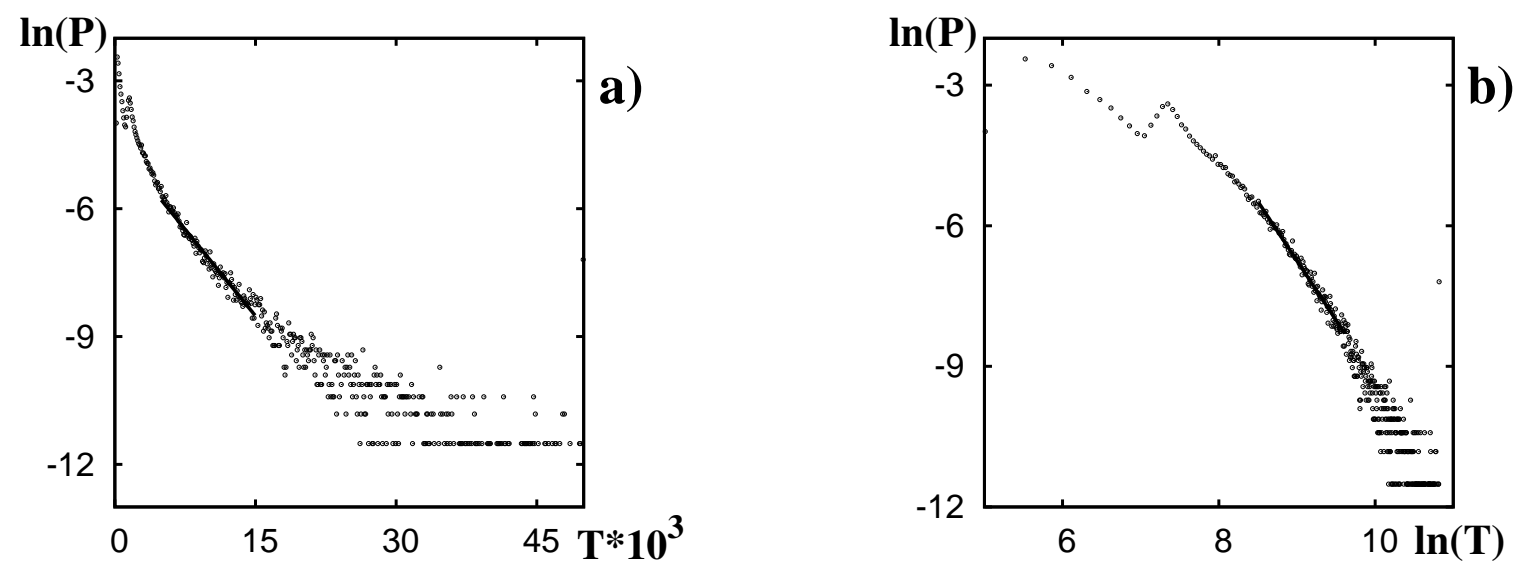

Figure 6: The probability distribution function for exit times $T$ in (a) semilogarithmic scale (exponential decay in the middle part with the exponent $\alpha=-0.000270722$ ) and (b) logarithmic scale (power-law decay at the tail with the coefficient $\gamma=-2.53086)$.

[3] S.V. Prants, Hamiltonian chaos with a cold atom in an optical lattice. In book: Hamiltonian Chaos beyond the KAM Theory. (Editors:A.C.J. Luo and N. Ibragimov) (Springer Verlag and Beijing: Higher Education Press, Berlin, 2010), 193-223.

[4] S. V. Prants and L.E. Kon'kov, JETP Letters, 73, 1801 (2001) [Pis'ma ZhETF, 73, 200 (2001)].

[5] S.V. Prants and V.Yu. Sirotkin, Phys. Rev. A, 64, 033412 (2001).

[6] S.V. Prants, JETP Letters, 75, 651 (2002) [Pis'ma ZhETF, 75, 777 (2002)].

[7] V. Yu. Argonov and S. V. Prants, JETP, 96, 832 (2003) [ZhETF, 123, 946 (2003)].

[8] S. V. Prants and M. Yu. Uleysky, Phys. Lett. A, 309, 357-362 (2003).

[9] S.V. Prants, M.Yu. Uleysky, and V.Yu. Argonov, Phys. Rev. A, 73, art. 023807 (2006).

[10] V. Yu. Argonov and S. V. Prants, Phys. Rev. A, 75, art. 063428 (2007).

[11] S. V. Prants, M. Edelman, and G. M. Zaslavsky, Phys. Rev. E, 66, art. 046222 (2002).

[12] V. Yu. Argonov and S. V. Prants, J. Russ. Laser Res., 27, 360 (2006).

[13] V. Yu. Argonov and S. V. Prants, Phys. Rev. A, 78, art. 043413 (2008).

[14] V.Yu. Argonov and S.V. Prants, Europhys. Lett., 81, art. 24003 (2008).

[15] M. Ben Dahan, E. Peik, J. Reichel, Y. Castin, and C. Salomon, Phys. Rev. Lett., 76, 4508 (1996).

[16] M.C. Fischer, K.W. Madision, Q. Niu, and M.G. Raizen Phys. Rev. A, 58, R2648 (1998).

[17] L.E. Kon'kov and S. V. Prants, J. Math. Phys., 37, 1204 (1996). 
[18] L.E. Kon'kov and S. V. Prants, JETP Letters, 65, 833 (1997) [Pis'ma ZhETF, 65, 801 (1997)].

[19] P. Gaspard, Chaos, Scattering and Statistical Mechanics, (Cambridge University Press, Cambridge, 1998).

[20] J. Aguirre, R.L. Viana, and M.A.F. Sanjuan, Rev. Mod. Phys., 81, 333 (2009).

[21] J.M. Petit and M. Henon, Icarus, 60, 536 (1986).

[22] M. Budyansky, M. Uleysky, and S. Prants, Physica D, 195, 369 (2004).

[23] M.V. Budyansky, M.Yu. Uleysky, and S.V. Prants, JETP, 99, 1018 (2004) [ZhETF, 126, 1167 (2004)].

[24] D.V. Makarov, M.Yu. Uleysky, and S.V. Prants, Chaos, 14, N1 79-95 (2004).

[25] M. Glück, A.R Kolovsky, and H.J. Korsch, Phys. Rep., 366, 103 (2002).

[26] B. Eckhardt, Physica D, 33, 89 (1988).

[27] G.M. Zaslavsky Hamiltonian chaos and fractional dynamics (Oxford: University Press, Oxford, 2005), 421.

[28] S.V. Prants, M.V. Budyansky, M.Yu. Uleysky, and G.M. Zaslavsky, Chaos, 16, art. 033117 (2006). 